\title{
Identificación y valor nutricional de algunos materiales nativos de arándano (Vaccinium spp).
}

\author{
Identification and nutritional value of some wild \\ materials of blueberry (Vaccinium spp).
}

Vilma Jiménez-Bonilla' Ana Abdelnour-Esquivel ${ }^{2}$ 


\section{Palabras clave}

Vaccinium; Ericaceae; arándano; antioxidantes; valor nutricional.

\section{Resumen}

El arándano es un arbusto de la familia de las ericáceas del género Vaccinium. Sus frutos son bayas de color oscuro, azuladas o rojizas. Presenta cualidades nutricionales $y$ antioxidantes que lo hace un fruto de alto valor medicinal y nutricional. En el curso de este estudio se localizaron, recolectaron, identificaron y analizaron por el método de ORAC (capacidad antioxidante total) varios materiales silvestres identificados como Vaccinium consanguinium. Los análisis químicos mostraron los altos índices de antioxidantes presentes en los frutos (Las Torres I, I 31,58; Las Torres 2, 156,20; Villa Mills II 2,84 y Quetzales

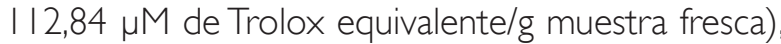
valores mayores a los indicados en la literatura para alimentos reconocidos por su potencial antioxidante. Debido al alto valor nutricional de los arándanos silvestres mostrado en este estudio, se recomienda incentivar y apoyar los esfuerzos nacionales para su domesticación y posterior siembra comercial.

\section{Key words}

Vaccinium; Ericaceae; blueberry; antioxidant; nutritional value.

\section{Summary}

The blueberry is a shrub of the family Ericaceae, genus Vaccinium. Its fruits are berries of dark, bluish or reddish coloration. Presents nutritional qualities and antioxidants which make it a fruit of high nutritional and medicinal value. During this study several wild materials identified as Vaccinium consanguinium were located, collected, identified and analyzed by the method of ORAC (antioxidant total capacity). Chemical analysis showed high levels of antioxidants in the fruits ((LasTorres I 131.58, Las Torres2 156.20,

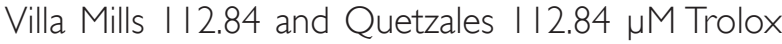
equivalent/g fresh fruits), values greater than those specified in the literature for food recognized by its antioxidant potential. Due to high nutritional value of wild blueberry showed on this study, it is recommended to encourage and support national efforts for their domestication and subsequent commercial planting.

\section{Introducción}

El arándano es un arbusto de la familia de las Ericáceas, género Vaccinium. Sus frutos son bayas de color oscuro, azuladas o rojizas, ricas en antocianos y minerales. Sus cualidades nutricionales y antioxidantes lo hace un fruto de alto valor medicinal y nutricional. En la alimentación humana, el arándano constituye una de las fuentes más importantes de antocianos y carotenoides, que le confieren su color característico y propiedades antioxidantes.

Estos frutos poseen un bajo valor calórico, son ricos en vitamina $C$, potasio, hierro y calcio, el cual es necesario en la transmisión y generación del impulso nervioso, en la actividad muscular normal e intervienen además en el equilibrio de agua dentro y fuera de la célula. Además, los frutos constituyen una buena fuente de fibra que mejora el tránsito intestinal. También contienen taninos, los cuales les confieren propiedades astringentes. La principal propiedad de estos frutos son sus altos contenidos de antocianos y vitaminas, que intervienen en la formación de colágeno, huesos, dientes, glóbulos rojos y favorecen la absorción del hierro de los alimentos y la resistencia a las infecciones (EROSKI, s.f.; Ostrolucka et al., 2008).

Los compuestos antioxidantes del arándano son flavonoides y ácidos fenólicos y los flavonoides se mencionan como antioxidantes particularmente poderosos (INTA 20ll). Los pigmentos naturales, junto con ácidos orgánicos como el oxálico y el málico, son los responsables de su sabor. En 
general, los antioxidantes pueden ayudar a proteger de enfermedades como cáncer, cardiovasculares y cerebrovasculares y arterioesclerosis (Ostrolucka et al., 2008). Una abundante evidencia indica que el efecto dañino de los radicales libres en lípidos, proteínas y ácidos nucleicos puede ser neutralizado por los antioxidantes y debido a que las frutas $y$ vegetales contienen diferentes compuestos y niveles de antioxidantes, el interés por determinar y comparar sus niveles se ha convertido en un área de interés.

Existen varios métodos para determinar la capacidad antioxidante de un alimento. Entre ellos se mencionan el TEAC (Tolox Equivalente Antioxidant Capacity, DPPH (2,2-Difenil-I-picrilhidrazil) y el ORAC (Oxygen Radical Absorbance Capacity o Capacidad de Absorción de Radicales de Oxígeno). Sin embargo, hay consenso en que para caracterizar la actividad antioxidante de un alimento, el ensayo ORAC se destaca por su alta sensibilidad, precisión y reproductividad, ya que permite medir la actividad o capacidad global que tienen todos los antioxidantes presentes en una muestra para neutralizar radicales peróxido, es decir, mide el aporte que hacen a la capacidad antioxidante tanto los polifenoles como aquellos compuestos de naturaleza no fenólica, lo cual permite comparar alimentos de naturaleza muy diversa en cuanto a su capacidad antioxidante (INTA, 20I I).

El arándano se menciona como una de las frutas con mayores contenidos de antioxidantes y de tipo flavonoide, lo que ha estimulado su consumo a escala mundial. En Costa Rica, a pesar de la creencia de que esta fruta es foránea, hay varias especies del género Vaccinium presentes en el territorio nacional que se distribuyen en un rango altitudinal de 1500 3500 metros sobre el nivel del mar (msnm), en los bosques montanos de la cordillera de Talamanca y en los alrededores del volcán Irazú. En nuestro país, el arándano es una de las especies silvestres cuya domesticación ha despertado gran interés, ya que es horticulturalmente promisorio (Montero, A. 2010, Gerente del Programa Nacional de Frutas No Tradicionales del Ministerio de Agricultura y Ganadería, comunicación personal). Este cultivo tiene gran potencial para la exportación y la industrialización a nivel nacional. Además, el país cuenta con suelos y climas favorables para su cultivo (Madriz, 1999). Por estas razones, el arándano se incluyó como línea de investigación en el área de cultivos no tradicionales del Programa Nacional de Fruticultura (Montero, A. 2010, comunicación personal).

Desde hace algunos años, los principales supermercados del país importan este fruto procesado en diferentes presentaciones (jugos, mermeladas, toppings, pasteles y otros) y más recientemente como fruta congelada. PROCOMER (2012) señala que los frutos de Vaccinium se importan desde Chile, Colombia, México y Estados Unidos y que las divisas invertidas para ello se han incrementado en los últimos años, pasando de US\$48.000 en 2007 a US\$103.000 en 20 I I, lo que demuestra el aumento en el consumo nacional de arándanos. Sin embargo, por su alto precio en el mercado local, su consumo probablemente está limitado a un sector selecto de la población.

El alto costo de importación, aunado a la presencia de varias especies silvestres de arándanos en el país de las cuales no se dispone de información -y sobre todo no se conocen sus cualidades nutricionales y antioxidantes, lo que permitiría compararlas con las especies que se comercializan- hace urgente este tipo de estudios, ya que si resultaran valiosas en estas características se podría iniciar el proceso de domesticación para cultivarlas y ofrecer una nueva opción de producción y comercialización a los productores nacionales. Por lo anterior, este estudio se propuso identificar y determinar el valor nutricional de algunas especies nativas de arándano (Vacciniun spp.) que presentaran un tamaño y color comparables a los de las frutas que se encuentran en el mercado.

\section{Materiales y métodos}

Durante el desarrollo del estudio se realizaron inicialmente revisiones bibliográficas para conocer con más exactitud la distribución geográfica de estas plantas en Costa Rica (INBIO, 20I0). Con base en esta información, la accesibilidad de los sitios y el presupuesto disponible, se eligieron los sitios que se visitarían para explorar el material, conocer su tamaño, forma y color. Los sitios visitados se ubican en la zona norte de Cartago, el volcán Poás, la Carretera Interamericana y el Cerro de la Muerte (Área de Conservación la Amistad, Ojo de Agua, Madre Selva, El Empalme, Villa Mills), Los Santos y el volcán Turrialba. Las giras se iniciaron en el mes de abril de 201 I y se mantuvieron periódicamente durante 
el resto del año, ya que las plantas localizadas en dichos lugares se encontraban en crecimiento vegetativo, por lo que fue imposible inicialmente lograr la identificación taxonómica ya que las estructuras reproductivas son básicas para este propósito. Por lo anterior se procedió a marcarlas y continuar con las visitas a estos mismos lugares en la época de fructificación. Con base en las características del fruto (tamaño y grado de madurez), se seleccionaron los materiales para analizar químicamente. Se marcaron los arbustos con cintas de colores, se tomaron muestras de ramas, flores y frutos y se colocaron en bolsas plásticas debidamente rotuladas. Para tener más certeza durante la colecta e identificación de las plantas, se solicitó la cooperación y el acompañamiento en las giras del MSc. Joaquín Sánchez González, botánico experto en el género, funcionario del Herbario del Museo Nacional, sitio al que se llevaron todas las muestras recolectadas en los diferentes sitios.

Una vez alcanzado el estado de maduración, con su típica coloración azul rojizo, se recolectaron $30 \mathrm{~g}$ de frutos por árbol muestreado (cinco árboles por sitio) y se llevaron al Laboratorio Físico-Químico del Centro de Investigación en Tecnología de Alimentos de la Universidad de Costa Rica, donde se les realizaron los análisis nutricionales. El método utilizado fue el ORAC. Los resultados tardaron cinco semanas para ser emitidos, por disposiciones protocolarias del Laboratorio.

\section{Resultados y discusión}

En los sitios mencionados en la literatura como hábitat de este género (INBio, 20I0) no se localizaron en este estudio plantas de Vaccinium (arándano), ni en la zona norte de Cartago ni en los alrededores de los volcanes Poás y Turrialba. Gran parte del material que se encontró abundantemente en estas zonas, así como en otras visitadas, pertenecía a las familias Ericaceae y Myrtaceae, conocidos comúnmente como arrayanes, turruses y otros nombres que popularmente también se le dan a los arándanos silvestres, lo que creó confusión en cuanto a la localización de los verdaderos arándanos. Esta situación, sumada a que en las etapas vegetativas estos materiales no se pueden identificar con certeza, aumentó la dificultad de su reconocimiento, ya que en el fruto se encuentra la característica taxonómica más importante para identificarlo a nivel de género y especie. Las plantas de la familia Ericaceae pueden presentar especies con ovario súpero o ínfero, mientras que el fruto del arándano tiene ovario súpero (Montiel, 1991); los otros materiales predominantes en las zonas visitadas presentaron ovario ínfero, lo que dificultó la identificación certera de los arándanos antes de su fructificación. Para identificar las diferencias a nivel de especies en este género se debió contar con material reproductivo, como flores y frutos, y en el momento de las primeras giras la mayoría de los arrayanes no los tenían. La aparición de flores y frutos se presentó a partir de agosto y septiembre y algunos de los frutos maduraron hasta octubre y diciembre.

A pesar de que la literatura menciona que en las zonas visitadas en este estudio se encuentran varias especies de arándano (Madriz, 1999), todos los materiales recolectados se identificaron como Vaccinium consanguineum (MSc. Joaquín Sánchez González, Herbario Nacional, comunicación personal). Los materiales identificados como del género Vaccinium fueron únicamente los recolectados en la Carretera Interamericana y el Cerro de la Muerte (Cuadro I).

En el análisis químico, las cuatro muestras identificadas como arándano (Vaccinium consanguineum), recolectadas en el Cerro de la Muerte y la Carretera Inteamericana (Las Torres I y 2, Villa Mills y Quetzales) mostraron altos índices ORAC, lo que significa un alto contenido de sustancias antioxidantes totales. Aunque las muestras son de la misma especie, se pudieron observar diferencias en los valores (entre I I2,84 y |56,20 $\mu$ M de Trolox equivalente/g muestra fresca), lo que podría indicar que son diferentes ecotipos o que las muestras presentaron diferente grado de maduración a la hora de la cosecha, sin que se pudieran observar estas diferencia a simple vista. También se determinó el índice ORAC en otro arrayán de la familia Ericaceae (Pernettya coriacea). Esta planta mostró un alto contenido de antioxidantes totales ( $122,10 \mu \mathrm{M}$ de Trolox equivalente/g muestra fresca), cuyo fruto y planta, en general, son muy similares a los arándanos, lo que podría explicar la confusión popular con los arándanos (Cuadro I).

En general, el poder antioxidante de todos los materiales de arándano analizados fue alto (Cuadro I). Al confrontar los valores ORAC de los arándanos con el arrayán (Pernettya coriacea) (Cuadro I), se 
Cuadro I. Valores de antioxidantes totales (análisis ORAC con base en peso fresco y peso seco) en muestras de arándano (Vaccinium consanguineum) y Pernettya coriacea de diferentes localidades de Costa Rica.

\begin{tabular}{|c|c|c|c|}
\hline Muestra & Humedad \% & $\begin{array}{c}\text { ORAC } \\
\text { ( } \mu \text { M de Trolox equivalente/g } \\
\text { muestra fresca) }\end{array}$ & $\begin{array}{c}\text { ORAC } \\
\text { ( } \mu \text { M de Trolox equivalente/g } \\
\text { muestra seca) }\end{array}$ \\
\hline Las Torres I & 76,8 & 131,58 & 564,6 \\
\hline Las Torres 2 & 76,9 & 156,20 & 676,19 \\
\hline Villa Mills & 74,8 & 144,50 & 573,4 \\
\hline Quetzales & 79,3 & 112,84 & 545,12 \\
\hline Pernettya coriacea & 70,2 & 122,10 & 409,73 \\
\hline
\end{tabular}

pudo observar que son muy similares, sobre todo a la muestra de Las Torres I (122,10 y 131,58 $\mu \mathrm{M}$ de Trolox equivalente/g muestra fresca) respectivamente.

Por otra parte, la literatura reporta valores ORAC para muchos de los principales alimentos reconocidos por su efecto antioxidante (Wang et al., 1996). En infusiones de café se indica un valor de 1,53 con base en peso seco, lo que es muy inferior al de los arándanos silvestres (Murthy y Madhava, 20I0). Además, los valores ( $\mu \mathrm{M}$ de Trolox equivalente/g muestra fresca) reportados en ciruela (73), mora (53), frambuesa (48), manzana (43) y naranja (18), entre otros, muestran valores muy inferiores a los determinados en los materiales silvestres analizados en este estudio. El único fruto reportado que supera en gran mediada el contenido de antioxidante de los arándanos silvestres es el fruto del acai (Euterpe oleracea), especie perteneciente a la familia de las palmeras, cultivada en Brasil por sus frutos y corazones de palmito (INKANATURAL, 2008).

Es importante resaltar los índices de actividad antioxidante mostrados por los arándanos que se comercializan en el país (94 y $92 \mu \mathrm{M}$ de Trolox equivalente/g muestra fresca), que son menores a los arrojados por los análisis de los arándanos silvestres de este estudio (INKANATURAL, 2008). Estas comparaciones ofrecen importante información acerca del potencial antioxidante de las especies silvestres de arándano de nuestro país y, con base en los resultados obtenidos en este estudio, se justifica incentivar y apoyar las iniciativas de domesticar estas plantas para introducirlas en la producción comercial.
Después de analizar los resultados del presente estudio se puede concluir, sin lugar a dudas, que el arándano silvestre (Vaccinium consanguinium) presenta un alto valor nutricional, ya que sus índices de antioxidantes totales superan por mucho a diferentes frutales reconocidos como ricos en estas sustancias.

Por todo lo anterior, es recomendable desarrollar metodologías para su propagación vegetativa, principalmente evaluar la técnica de micropropagación, ya que permite la propagación acelerada y masiva de los materiales de interés, a diferencia de las técnicas tradicionales de propagación o de la propagación sexual, que presenta dificultades, lo que permitiría hacer pruebas de su comportamiento en diferentes sitios de siembra y desarrollar su manejo agronómico.

\section{Agradecimientos}

Las investigadoras agradecen el apoyo financiero de la Vicerrectoría de Investigación y Extensión del Instituto Tecnológico de Costa Rica. Al MSc. Joaquín Sánchez González, botánico del Herbario del Museo Nacional, por su colaboración en la identificación de los materiales, y a la MSc. Patricia Arguedas, por su apoyo en la interpretación de los análisis químicos.

\section{Bibliografía}

EROSKI, s.f. Frutas sabrosas, saludables, imprescindibles. En: http// frutas.Consumer.es/documentos/arándano. (Consultado 23 abril 2011 )

INBio (Instituto Nacional de Biodiversidad) (2010). Lista de Especímenes de Vaccinium spp. Disponible en: http://www. 
inbio.ac.cr/bims/k03/p I 3/c045/o0 I 593/g0076 I 5/s022638. htm (Consultado febrero 201 I).

INKANATURAL (2008). Acai: Fruto amazónico para dieta. Disponible en: www.inkanatural.com/es/arti.asp (Consultado febrero 2012).

INTA (Instituto Nacional de Nutrición y Tecnología de Alimentos) (20 I I). Análisis de antioxidantes: Qué y cómo se deben medir. F: Arandano\ANÁLISIS DEANTIOXIDANTES QUÉ Y CÓMO SE DEBE MEDIR Antioxidantes Portal Antioxidantes Primer Portal de Antioxidantes, Alimentos y Salud en el Mundo de Habla Hispana.mht (Consultado 25 octubre 201 I).

Madriz, J.P. (1999). Vaccinium: Especies silvestres neotropicales, perspectivas para la domesticación de nuevos frutales arbustivos, en los bosques montanos del neotrópico. Memorias IX Congreso Nacional Agronómico. p. 295.
Montiel Longhi, M. (|99|). Introducción a la flora de Costa Rica. 2da. ed. Editorial Universidad de Costa Rica. p. 25I.

Murthy, S. \& Madhava, M. (2010). Recovery of Phenolic Antioxidants and Functional Compounds from Coffee Industry By-Products. Food Bioprocess Tecnol. Publicado en línea por Springer.

Ostrolucka, M., Gajdosova, A., Libiakova, G., Hrubikova, K. \& Bezo, M. (2007). Protocol for micropropagation of selected Vaccinium spp. En: Jain, S.M., Haggman, H. (ed.). Protocols for micropropagation of Woody Trees and Fruits. Springer, p. 445-455.

PROCOMER (Promotora de Comercio Exterior). (20/2). En: http://www.procomer.com/contenido/informaci\%C3\%B3nde-mercados-y-sectores-html. (Consultado 20 de enero 2012).

Wang, H., Cao, G. \& Prior, R. (1996). Total antioxidant capacity of fruits. J. Agric. Food Chem. 44: 70I-705. 